\title{
Preferential recognition and antagonism of SARS-CoV-2 spike glycoprotein binding to 3-O-sulfated heparan sulfate
}

\begin{abstract}
Vaibhav Tiwari, ${ }^{* 1}$ Ritesh Tandon, ${ }^{2}$ Nehru Viji Sankaranarayanan, ${ }^{3}$ Jacob C. Beer, ${ }^{1}$ Ellen K. Kohlmeir, ${ }^{4}$
\end{abstract} Michelle Swanson-Mungerson ${ }^{1}$ and Umesh R. Desai*3

${ }^{3}$ Department of Medicinal Chemistry and Institute for Structural Biology, Drug Discovery and Development, Virginia Commonwealth University, 800 E. Leigh Street, Suite 212, Richmond, VA 23219

${ }^{1}$ Department of Microbiology and Immunology, Midwestern University, Downers Grove, IL 60515

${ }^{2}$ Department of Microbiology and Immunology, University of Mississippi Medical Center, Jackson, MS 39216

${ }^{4}$ Core Facility, Midwestern University, Downers Grove, IL 60515

*Corresponding Authors: V. Tiwari (vtiwar@midwestern.edu) \& Umesh R. Desai (urdesai@,vcu.edu)

Running title: SARS-CoV-2 Spike - 3-O-sulfated HS interaction

KEYWORDS: Coronavirus, Heparan Sulfate, Small Molecule Inhibitor, Spike glycoprotein, Antagonists 
Abstract: The COVID-19 pandemic caused by SARS-CoV-2 is in immediate need of an effective antidote. Although the Spike glycoprotein $(\mathrm{SgP})$ of SARS-CoV-2 has been shown to bind to heparins, the structural features of this interaction, the role of a plausible heparan sulfate proteoglycan (HSPG) receptor, and the antagonism of this pathway through small molecules remain unaddressed. Using an in vitro cellular assay, we demonstrate HSPGs modified by the 3-O-sulfotransferase isoform-3, but not isoform-5, preferentially increased $\mathrm{SgP}$-mediated cell-to-cell fusion in comparison to control, unmodified, wild-type HSPGs. Computational studies support preferential recognition of the receptor-binding domain of $\mathrm{SgP}$ by $3-O$-sulfated HS sequences. Competition with either fondaparinux, a 3-O-sulfated HS-binding oligopeptide, or a synthetic, non-sugar small molecule, blocked SgP-mediated cell-to-cell fusion. Finally, the synthetic, sulfated molecule inhibited fusion of GFP-tagged pseudo SARSCoV-2 with human 293T cells with submicromolar potency. Overall, overexpression of 3-O-sulfated HSPGs contribute to fusion of SARS-CoV-2, which could be effectively antagonized by a synthetic, small molecule.

\section{Introduction}

The 2019 novel coronavirus (2019-nCoV, official name: SARS-CoV-2), the causative agent behind the current pandemic, is proving to be highly lethal. SARS-CoV-2 is a member of the family of coronaviruses that generally cause routine infections in humans; however, the severity of organ failure, especially the lung, caused by this virus necessitates studies on all molecular pathways that may be targeted for intervention (1). Of these, virus attachment and internalization pathways are the key to devising strategies that prevent infection even in the early or asymptomatic phase.

The cellular entry of SARS-CoV-2 has been shown to depend on the binding of the viral spike glycoprotein $(\mathrm{SgP})$ to host cell ACE-2 receptor (2-4). Whereas the SgP-ACE-2 pathway has been the focus of most studies, host cell surface heparan sulfate proteoglycans (HSPGs) have also been shown to play important roles in pathology of enveloped viruses, e.g., coronaviruses, herpes simplex virus (HSV), cytomegalovirus, dengue virus, and hepatitis E virus (5-9). In fact, the Esko group has recently shown that infectivity of the SARS-CoV-2 depends on cell surface HSPGs (10). Further, evidence has also been presented that the molecular diversity of HS chains plays an important role in supporting entry, trafficking and replication processes (5), which span a majority of the cellular processes in the life cycle of the virus.

HSPGs contain one or more heparan sulfate (HS) chains covalently linked to serine residues of a core protein such as syndecan and glypican (11). HS is made up of alternating $D$-glucuronic acid (plus some $L$-iduronic acid) and $N$ acetylglucosamine residues that are variably modified by a cascade of sulfotransferases (STs) that engineer sulfated microdomains along the polymeric chain. These microdomains form unique sites of binding for different cell surface receptors, soluble proteins and enzymes $(12,13)$.

A classic ST is the 3-O-sulfotransferase, for which six different isoforms (i.e., 3OST-1, -2, $3_{\mathrm{A}},-3_{\mathrm{B}},-4$ and -5$)$ are known (14). Each of these 3OSTs exhibit subtle differences in substrate specificities, thereby engineering rare sulfation microdomains or recognition "codes" in HSPGs (15). While these 3-O-sulfate "codes" are significantly different from the common, non-3$O$-sulfated regions on the HS biopolymer, the selectivity of protein recognition between the multiple 3-O-sulfate "codes" may not necessarily be exquisite, as exemplified by the observation that all 3OSTs, except for 3OST-1 (16), support HSV-1 entry and spread (17-21).

Based on the role of HSPGs in viral adherence and internalization $(1,21)$, we hypothesized that SARS-CoV-2 may exhibit subtle role of HSPG microstructure, i.e., the local microdomains within $\mathrm{HS}$, to advantageously gain entry into a host cell. Here, we demonstrate using a model cellular cell-to-cell fusion assay that the $\mathrm{SgP}$ of SARS-CoV-2 demonstrates better recognition of 3-OST-3 ${ }_{\mathrm{B}}$-modified heparan sulfate (HS) receptor in comparison to either the wild-type, unmodified HSPG or the 3OST-5 
modified HSPG. Computational studies offer a structural foundation to this role as originating from selective binding of 3- $O$-sulfated sequences to the $\mathrm{SgP}$. More importantly, this pathway could be antagonized by deploying specific agents such as fondaparinux, an anti-3-O-sulfated-HS peptide or a synthetic non-sugar, sulfated, small molecule. Overall, this work provides critical recognition and antagonism insights that should assist developing therapeutics against SARSCoV-2 attachment and internalization, which could rapidly reduce infection rates.

\section{Results and Discussion}

To assess whether HSPGs mediate cell fusion with an SgP-bearing cell, a luciferase reporter gene activation assay was used in Chinese hamster ovary (CHO-K1) cells, as described in our work $(17,22,23)$. We selected CHO-K1 cells for our cell fusion experiment because these cells lack functional surface receptors for SARS-CoV including human ACE-2 (24). This makes them resistant to infection. Further, CHO-K1 cells are also known to lack endogenous expression of 3OSTs, which implies that their HSPGs do not carry 3-O-sulfated microdomains (5). In combination, $\mathrm{CHO}-\mathrm{K} 1$ cells provide an excellent platform to identify new receptor targets that may support SARS-CoV-2 entry in absence of ACE2.

The cell susceptibility of SARS-CoV-2 being probed here utilizes a cell-to-cell fusion model in which fusion between "effector" and "target" CHO-K1 cells results in activation of the luciferase gene, which is quantified $24 \mathrm{~h}$ post coculture using luminescence spectrophotometry. The "effector" cells are transiently co-transfected with plasmids expressing the SARS-CoV-2 SgP and T7 polymerase, while the "target" cells are co-transfected with plasmids carrying either human 3 OST- 3 B, 3-OST-5 or ACE-2 genes along with luciferase expression plasmid. As wild-type negative controls, the "target" CHO-K1 cells are co-transfected with an empty vector (pCAGGS), devoid of the 3 OST- $3_{\mathrm{B}}, 3-\mathrm{OST}-5$ or ACE-2 genes, whereas the positive control "target" cells expressed only the human ACE-2 receptor (see Supplementary Materials).
The advantage of this in vitro model system is that it affords direct insight into structural features of the SgP-HSPG interaction in cellular settings without the complexities that abound full virus studies. It specifically provides information on receptors other than ACE-2, which may facilitate virus internalization/fusion, especially with regard to modified forms of HS. It is important to note that this cell-to-cell fusion assay is not directed to conclude on the mode of SARS-CoV-2 entry, i.e., membrane fusion or endocytosis, which varies depending on the type of cell (25).

As shown in Figure 1A, the "target" negative control CHO-K1 cells expressing wild-type HS displayed only marginal fusion with "effector" CHO-K1 cells expressing SgP. In contrast, nearly 3 -fold higher fusion was detected in the presence of either ACE-2 and/or 3-O-sulfated HS receptors. This suggests a critical role for the 3OST-3 $3_{\mathrm{B}}$ modified HSPG receptor in SgPmediated cell-to-cell fusion during viral spread. It is important to note that $3 \mathrm{OST}-3_{\mathrm{B}}$-modified HSPG "target" cells do not contain the wellestablished SARS-CoV-2 receptor ACE-2 (2628). Further, transfecting varying levels of $3 \mathrm{OST}$ $3_{\mathrm{B}}$ plasmid alone into the effector cells led to a concomitant increase in cell-to-cell fusion (see Figure S1). Thus, the results show that SgPmediated cell-to-cell fusion arises even in the absence of ACE-2.

Interestingly, no statistical difference in cellto-cell fusion between $\mathrm{CHO}-\mathrm{K} 1$ cells carrying either ACE-2 or 3OST-3 $\mathrm{B}$ HSPG receptors was observed (Figure 1A). Additionally, the combined presence of ACE-2 and 3-O-sulfated HSPG did not exhibit any additivity or synergism under the conditions studied. Although this suggests saturation of cell-to-cell fusion with either 3-O-sulfated HSPGs or ACE-2-mediated pathways, detailed studies would be needed to ascertain absence of synergism under all conditions.

The SgP of SARS-CoV-2, through specific interactions with the host cell receptor, is also known to contribute to syncytia formation (29), an important pathway in the pathogenesis of the virus. Therefore, we next assessed syncytia formation by co-culturing $\mathrm{SgP}$ expressing 
"effector" cell with "target" CHO-K1 cells expressing HSPGs or ACE-2. As shown in the Figure 1B, minimal (or no) syncytia formation was observed for target cells carrying wild-type HSPG receptor (Panel 'WT-HS'), while much higher number of syncytia were observed in SgPmediated fusion with target cells carrying either ACE-2 (Panel 'ACE-2') or HSPGs modified by $3 \mathrm{OST}-3_{\mathrm{B}}$. Here, co-expression of both $3 \mathrm{OST}-3_{\mathrm{B}}$ and ACE-2 displayed higher syncytia numbers. Taken together, these results ascertain that 3-Osulfated HSPGs are likely to play an important role in SgP-mediated cell-to-cell fusion process.

We next assessed selectivity of 3OSTmodified sub-domain recognition by SARSCoV-2 SgP by comparing target CHO-K1 cells transfected with plasmids expressing either 3 OST $-3_{\mathrm{B}}$ or 3 OST -5 . The results showed that cells expressing $3 \mathrm{OST}-3_{\mathrm{B}}$ displayed $\sim 3$-fold more cell-to-cell fusion than 3OST-5 expressing cells (Figure 1C). In fact, fusion with 3OST-5 effector cells was very similar to the negative control, wild-type HSPG-carrying effector cells. This implies that SARS-CoV-2 SgP differentially recognizes the 3-O-sulfated HS structures generated by the two different isoforms, 3OST$3_{\mathrm{B}}$ and 3OST-5. This result is strikingly different from HSV-1, which can utilize both 3OST-3 and 3OST-5 isoform-generated HSPGs for cell fusion (17). Thus, it appears that SgP of SARS-CoV-2 is more selective in its recognition of the HSPG receptor. A quick question to address here is whether differential expression of 3OST-3 and 3OST-5 could have contributed to differential recognition of $\mathrm{SgP}$. To mitigate this possibility, we performed simultaneous experiments for HSV-1 entry. Our results suggested that cells expressing both $3 \mathrm{OST}-3_{\mathrm{B}}$ and $3 \mathrm{OST}-5$, but not the control cells (i.e., containing unmodified HSPGs), allowed HSV-1 entry $(5,17)$ (see Figure S2). Finally, we also tested whether more relevant human cells, i.e., human lung epithelial A549 cells, would exhibit 3-OST-3 $3_{B}$ enhanced $\mathrm{SgP}-$ mediated cell fusion. Figure $\mathrm{S} 3$ shows that $3 \mathrm{OST}-3_{\mathrm{B}}$ overexpression enhances fusion in the manner observed for CHO-K1 cells. Thus, SARS-CoV-2 SgP recognition of HSPGs is different from that HSV-1 glyroproteins.

To better understand $\mathrm{SgP}$ features that contribute to recognition of HSPGs and mediation of cell-to-cell fusion, we studied full length $\mathrm{SgP}$ as well as its receptor-binding domain (RBD) alone. Full length $\mathrm{SgP}$ contains two subunits, $\mathrm{S} 1$ and $\mathrm{S} 2$. The RBD is located within the $\mathrm{S} 1$ subunit $(2,31,32)$ and is known to mediate interaction with the ACE-2 receptor. In contrast, the S2 subunit contributes to cellular internalization $(33,34)$. Do these functions hold when 3-O-sulfated HSPG is the only receptor present on target cells?

To address this, the effector cells expressing either full length $\mathrm{SgP}$, the RBD alone or both were co-cultured with target cells expressing the 3OST- $3_{\mathrm{B}}$ modified HSPG or negative control target cells expressing wild-type HSPG. The results indicated that full length $\mathrm{SgP}$, but not RBD alone, promoted 3-O-sulfated HSPG pathway (Figure 1D). Alternatively, in the absence of the ACE-2 receptor on target cells, fusion with effector cells occurs only with fulllength $\mathrm{SgP}$ and is dramatically impaired with RBD alone. This implies that the $\mathrm{S} 2$ subunit is critical for cell-to-cell fusion mediated by the $\mathrm{SgP}-3-O$-sulfated $\mathrm{HSPG}$ pathway. More importantly, cell fusion was miniscule when the effector cells expressing RBD alone were exposed to wild-type HSPG target cells in comparison to moderate level of cell fusion for 3$O$-sulfated HSPG target cells (Figure 1D). A similar phenotype with no cell fusion was observed when ACE-2 target cells were cocultured with effector cells carrying RBD alone (see Figure S4). This implies that the RBD preferentially recognizes 3-O-sulfated microdomains generated by $3 \mathrm{OST}-3_{\mathrm{B}}$.

We next addressed the question whether this selectivity of $\mathrm{SgP}$ recognition originates from differences in atomistic interactions with different sequences of HS. Recent studies have shown that the selectivity of HS recognition can be predicted through rigorous a dual-filter computational algorithm that compare and contrast interactions of a large number of HS sequences binding to proteins (35). We built a library of 27,930 unique topologies possible for natural di-, tetra, and hexasaccharides of HS in a combinatorial manner. This library of HS sequences was studied for recognition of the $\mathrm{RBD}$ of $\mathrm{SgP}$ trimer. Each of the 27,930 topologies was docked in triplicate using a 
genetic algorithm-based using a well-established dual-filter algorithm (Figure 2A). All three plausible sites of HS binding on $\mathrm{SgP}$ trimer, proposed recently by Linhardt and coworkers (36), were studied (Figures 2B and S5). Of these, the RBD was found to be the most favored site for HSPG to interact (not shown).

The predicted poses of HS binding onto RBD were analyzed using two parameters corresponding to in silico affinity (GOLD score) and consistency of binding (i.e, root mean square deviation (RMSD)) as implemented in our studies on multiple protein-HS systems $(16,37,38)$. The analysis indicated that none of the $30 \mathrm{di}-$ or 900 tetra- sequences recognized $\mathrm{SgP}$ well (not shown). In contrast, 242 of the 27,000 hexasaccharide topologies were predicted to interact with the RBD with high affinity (Score $>100$, Figure 2C). Nearly $92 \%$ of these, or 223 unique topologies, contained at least one 3-Osulfated glucosamine residue. An unusual structural characteristic of this group was the preferred placement of the 3-O-sulfate group on either the $1^{\text {st }}$ or $3^{\text {rd }}$ residue from the non-reducing end (see Table S1). In fact, majority of HS sequences favored by $\mathrm{SgP}$ contained multiple 3$O$-sulfated residues suggesting a structural characteristic that is likely to be unique for $\mathrm{SgP}$.

Interestingly, two sequences displayed high affinity as well as high selectivity for RBD (Score $>120$, RMSD $<2.5 \AA$; Figure 2D) by forming strong ionic and hydrogen bonding interactions (see Figure S6). Residues common in both sequences included GlcNS3S6S, IdoA2S and GlcNAc6S, which engineered high selectivity of interaction (Figure 2E). Interestingly, a glucuronic acid is present on the non-reducing end of the 3-O-sulfated glucosamine in one sequence, which is not known to be generated by 3OST-5 (39). Thus, these computational studies afford strong atomistic foundation to the concept that the RBD of SgP preferentially recognizes 3$O$-sulfated microdomains of HS.

We reasoned that the selectivity of $\mathrm{SgP}$ for 3$O$-sulfated HS could offer a route to discovering antagonists of cell-to-cell fusion. To study this, we first tested whether treatment with bacterial heparinase I, which can partially degrade cell surface HSPGs, would decrease HS-dependent fusion of "target" CHO-K1 cells with SgPbearing "effector" cells. It is important to note that heparinase I prefers heparin as a substrate (40); however, HS is also known to be depolymerized. As shown in Figure 3A, heparinase I-treated target cells displayed $\sim 2$-fold cell-to-cell fusion. Next we considered competitive antagonism, which has a better potential in terms of therapeutics. Hence, we studied fusion between 3-O-sulfated HS-bearing "target" cells and SgP-containing "effector" cells in the presence of a generic peptide targeting common HS sequences (G1) and a specific peptide directed towards 3-O-sulfated HS (G2). The G1 and G2 peptides were developed earlier, using phage display library screening, as probes for studying HS selectivity against HSV entry and spread (17,21-23). As evident from Figure $3 \mathrm{~A}, 10 \mu \mathrm{M}$ dose of both peptides depressed cell fusion, as would be expected on the basis of competitive antagonism. However, a striking difference of nearly 2-fold was observed for cells bearing the 3-O-sulfated $\mathrm{HS}$ receptor in comparison to the wild-type HS receptor (Figure $3 \mathrm{~A})$.

To further test the selectivity of 3-O-sulfated HS recognition and antagonism, we utilized fondaparinux, a clinically used anticoagulant. Fondaparinux is a unique, synthetic pentasaccharide with a central 3-O-sulfated glucosamine residue. As shown in Figure 3B, the addition of fondaparinux inhibited $\mathrm{SgP}$-mediated cell-to-cell fusion with "target" cells expressing 3-O-sulfated HS in a dose-dependent manner. At nearly $100 \mu \mathrm{M}$ level, fondaparinux reduced cell fusion to the basal levels observed for the wildtype HS receptor. This result further confirms the role of 3-O-sulfated $\mathrm{HS}$ in mediating $\mathrm{SgP}$-based cell fusion.

The highly sulfated nature of the HS hexasaccharides identified through computational studies (Figures 2 and S6, Table $\mathrm{S} 1$ ), also led us to reason that a synthetic, small, non-sugar, highly sulfated compound, called SPGG, could serve as an effective inhibitor of cell-to-cell fusion mediated by $\mathrm{SgP}$ (Figure 4). SPGG has recently been identified as a highly promising pan-virus antagonist of cellular entry because it competes for viral glycoproteins, such as glycoprotein D of HSV, that are involved in 
recognition of cell surface HSPGs (41-44). We studied SPGG's effect on cell-to-cell fusion in CHO-K1 cells as well human HEK293T cells. As shown in Figures 4A and 4B, the synthetic agent SPGG reduced cell-to-cell fusion in both cell lines quite effectively. As the concentration of SPGG increased to $1.0 \mu \mathrm{M}$, cell-to-cell fusion for both cell lines decreased approximately $50 \%$. This suggested a strong possibility that SPGG may exhibit highly promising anti-SARS-CoV-2 potential.

To further assess the translatability of SPGG's anti-cell-to-cell fusion activity, we generated GFP-expressing pseudo-typed SARSCoV-2 particles (pLV-S) using a third-generation lentivirus-system (45). These particles express the SARS-CoV-2 SgP on their surface in the native form. Briefly, HEK293T cells were cotransfected with a $p L V$-eGFP (the GFP plasmid), psPAX2 (packaging plasmid) and either $p C A G G S-S$ (SARS-CoV-2 plasmid) or VSV-G (control plasmid) (see Supplementary Materials). Following $48 \mathrm{~h}$ of co-culture, the virus (or control) particles were harvested, quantified and used for infection of new HEK293T cells at dilutions of $10^{2}$ to $10^{7}$ so that virus titers were in the range of 20 to 100 GFP positive cells. Two treatment modalities were explored. In mode A), SPGG was pre-incubated with the pLV-S pseudovirus before infecting the target 293T cells. In mode B), the $293 \mathrm{~T}$ cells were first pretreated with SPGG and then challenged with the pLV-S pseudo-virus.

Figure 4C shows that SPGG severely impaired viral entry in a dose-dependent manner in both treatment modalities. Interestingly, the modality of treatment did not impact SPGG's inhibition potential. Most importantly, the inhibition potency of SPGG could be between 0.1 and $1.0 \mu \mathrm{M}$ (see 50\% inhibition line, Figure 4C). A comparison of GFP fluorescence of $293 \mathrm{~T}$ cells revealed significant decrease in internalized GFP-tagged virus particles in the presence of SPGG (panel (ii), Figure 4D). Overall, the synthetic agent SPGG was identified to be a promising inhibitor of pseudo-typed SARS-CoV2 entry into human $293 \mathrm{~T}$ cells.

\section{Conclusions}

In this study, we have provided the first evidence that 3-O-sulfated microdomains in HS chains of HSPGs, especially those generated by 3 OST $-3_{\mathrm{B}}$, but not necessarily by 3OST-5, offer preferential recognition of SgP of SARS-CoV-2. This recognition affords unique opportunities for development of antagonists that may prevent cellto-cell fusion and viral spread. Of the several antagonists studied in this work, the synthetic, highly sulfated small molecule SPGG was especially effective in reducing $\mathrm{SgP}$-mediated cell-to-cell fusion and syncytia formation. Considering that this pathway has been reported for human enteroids (46) and also serves as a mechanism to evade antibody neutralization (47), SPGG as a sub-micromolar antagonist of this pathway has major translational value.

The entry of SARS-CoV-2 into host cells is a multi-step process involving several molecular and cellular factors. Whereas optimal and timely functioning of each of these factors is needed for the virus to multiply and spread, $\mathrm{SgP}$ is an obligatory factor because of its key role in cell surface receptor engagement, without which no virus entry is possible (2). ACE-2 was identified as the first host cell surface receptor for SARSCoV-2 entry $(3,31,32)$. Yet, growing evidence points to HSPG as another receptor that the virus uses to anchor to the host cell (10). This work establishes that modified forms of HS, especially those produced by $3-\mathrm{OST}-3_{\mathrm{B}}$, greatly enhance cell fusion to facilitate host cell entry. This result is of major significance because it has been known that inflamed cells and tissues of the lung exhibit higher levels of 3-O-sulfated HS (48). This implies that the inflammatory storm induced by SARS-CoV-2, especially in the lung, is likely to be significantly aided by 3-O-sulfated HSPGs, thereby orchestrating a self-feeding, selfdestructive cycle with a detrimental outcome.

Our model studies with CHO-K1 "target" and "effector" cells show that 3-O-sulfated HS alone can promote cell-to-cell fusion (Figure 1A). Our cell-fusion assay is a focused attempt to identify receptors other than ACE-2, which was clearly evident from the results with the 3-O sulfated modified forms of HS. One advantage of this assay is that functional domains and amino 
acid residues in $\mathrm{SgP}$ can be identified relatively easily to understand the impact of mutational changes. Such studies may be very worthwhile since SARS-CoV-2 has shown variability in the SgPs $(2,49)$.

This suggests that as far as cell-to-cell fusion process (50) is concerned 3-O-sulfated HSPGs on host cells may act as functional receptors, independent of ACE-2. Alternatively, other forms of cellular entry, e.g., through fusogenic proteins, may require ACE-2. As evident in recent studies (10), HSPGs play supporting role in such processes.

This work provides a unique insight in $\mathrm{SgP}$ recognition of 3-O-sulfated $\mathrm{HS}$ and ACE-2 receptors. Our work shows that $3 \mathrm{OST}-3_{\mathrm{B}}$ modified HSPG, but not wild-type HSPG, mediates fusion with the RBD alone (Figure 1D). Interestingly, a recent study has shown that the heparin binding to the RBD is accompanied by conformational changes, which may play a role in cell entry (51). In striking contrast, cell fusion with RBD alone was not observed for the ACE-2 receptor (Figure $\mathrm{S} 4$ ). Rather, it is well established that fusion mediated by the ACE- 2 receptor requires cleavage of the full length $\mathrm{SgP}$ into $\mathrm{S} 1$ and S2 units $(2,3)$. This highlights the differentiating role of 3-O-sulfated microdomains of HS in $\mathrm{SgP}$ mediated cell-to-cell fusion, especially through the RBD. Further, this work highlights the importance of studying other 3OSTs to elucidate additional receptor requirements, if any.

It is important to note that the activity of HSPG modifying enzymes, e.g., 3OSTs, is dependent on the availability of optimal substrate sites within existing HS chains $(15,16,52)$. In fact, the activity of the cascade of sulfotransferases and their isoforms (NDST, 2OST and 6OST), would determine the generation of 3-O-sulfated microdomains. This implies that populations of patients carrying upregulated HS biosynthetic genes may be more susceptible to SARS-CoV-2 infection. Rigorous studies with samples from different types of patients would be needed to evaluate this deduction from our work.

Several studies have shown that heparin or heparin-like molecules bind $\mathrm{SgP}$ with high affinity. Studies on full length and low molecular weight heparins by the Linhardt group measured potencies in the picomolar range (36), whereas the Boons group have reported nanomolar potencies for both polymeric and oligomeric heparins (53). Such high potencies could be advantageously translated into potential drug candidates, as proposed by the Turnbull group in the form of a clinical-stage heparan sulfate mimetic (54). Suramin, a small molecule mimetic of heparin, has also been presented as an inhibitor of early steps of SARS-CoV-2 infection (55).

Our work greatly expands these possibilities to include 3-O-sulfate microdomain targeting peptides as well as synthetic, high sulfated SPGG. Likewise, fondaparinux, which has a 3-Osulfate group, is also a significant inhibitory agent. Of these, SPGG is particularly attractive because of its ease of synthesis, lack of cytotoxicity, and broad spectrum antimicrobial activity $(42,43,56)$. SPGG is also an inhibitor of coagulation factor XIa (56), which would simultaneously induce an anti-coagulant effect without major bleeding risk. Thus, use of SPGG in SAR-CoV-2 has the potential of addressing episodes of thrombosis observed in large number of severely ill patients (57).

Overall, our results provide the first evidence of a role of 3-O-sulfated HS in SARS-CoV-2 SgP mediated cell fusion and viral entry. HS clearly plays a role in SARS-CoV-2 pathogenesis; however, 3-O-sulfated HS, especially when overexpressed, greatly enhances cell fusion. This work lays the foundation for development of small molecule agents against SARS-CoV-2. A good number of HS mimetics are currently in clinical trials, especially against cancer. This work highlights the promise of such HS mimetics in treatment and prevention of SARS-CoV-2 too. 


\section{Acknowledgements}

We thank the computational resources made available to us through a grant from the National Center for Research Resources (S10 RR027411) to VCU.

\section{Funding}

This work was supported in part by grants from the NIH including HL107152, HL090586 and CA241951 (URD) and start-up funding to VT by Midwestern University.

Conflict of Interest: The authors declare no conflicts of interest with regard to this manuscript.

\section{References}

1. Tiwari, V., Beer, J. C., Sankaranarayanan, NV., Swanson-Mungerson, M., and Desai, U. R. (2020) Discovering small-molecule therapeutics against SARS-CoV-2. Drug Disc. Today. 25, 1535-1544.

2. Walls, A. C., Park, Y. J., Tortorici, M. A., Wall, A., McGuire, A. T., and Veesler, D. (2020) Structure, Function, and Antigenicity of the SARS-CoV-2 Spike Glycoprotein. Cell 181, 281-292.

3. Letko, M., Marzi, A., and Munster, V. (2020) Functional assessment of cell entry and receptor usage for SARS-CoV-2 and other lineage B betacoronaviruses. Nat. Microbiol. 5, 562-569.

4. Zhou, P., Yang, X. L., Wang, X. G., Hu, B., Zhang, L., Zhang, W., Si, H.-R., Zhu, Y., Li, B., Huang, C.-L., Chen, H.-D., Chen, J., Luo, Y., Guo, H., Jiang, R.-D., Liu, M.-Q., Chen, Y., Shen, X.-R., Wang, X., Zheng, X.-S., Zhao, K., Chen, Q.-J., Deng, F., Liu, L.-L., Yan, B., Zhan, F.-X., Wang, Y.-Y., Xiao, G.-F., and Shi, Z.-L. (2020) A pneumonia outbreak associated with a new coronavirus of probable bat origin. Nature 579, 270-273.

5. Shukla, D., Liu, J., Blaiklock, P., Shworak, N. W., Bai, X., Esko, J. D., Cohen, G. H., Eisenberg, R. J., Rosenberg, R. D., and Spear, P. G. (1999) A novel role for 3-O-sulfated heparan sulfate in herpes simplex virus 1 entry. Cell 99, 13-22.

6. Hilgard, P. and Stockert, R. (2000) Heparan sulfate proteoglycans initiate dengue virus infection of hepatocytes. Hepatology 32, 1069-1077.

7. de Haan, C. A., Li, Z., te Lintelo, E., Bosch, B. J., Haijema, B. J., and Rottier, P. J. (2005) Murine coronavirus with an extended host range uses heparan sulfate as an entry receptor. J. Virol. 79, 1445114456.

8. Kalia, M., Chandra, V., Rahman, S. A., Sehgal, D., and Jameel, S. (2009) Heparan sulfate proteoglycans are required for cellular binding of the hepatitis E virus ORF2 capsid protein and for viral infection. $J$. Virol. 83, 12714-12724.

9. Milewska, A., Zarebski, M., Nowak, P., Stozek, K., Potempa, J., and Pyrc, K. (2014) Human coronavirus NL63 utilizes heparan sulfate proteoglycans for attachment to target cells. J. Virol. 88, 13221-13230.

10. Clausen, T. M., Sandoval, D. R., Spliid, C. B., Pihl, J., Perrett, H. R., Painter, C. D., Narayanan, A., Majowicz, S. A., Kwong, E. M., McVicar, R. N., Thacker, B. E., Glass, C. A., Zhang, Y., Torres, J. L., Golden, G. J., Bartels, P. L., Porell, R. N., Garretson, A. F., Laubach, L., Feldman, J., Yin, X., Pu, Y., Hauser, B., Caradonna, T. M., Kellman, B. P., Martino, C., Gordts, P. L. S. M., Chanda, S. K., Schmidt, A. G., Godula, K., Leibel, S. L., Jose, J., Corbett, K. D., Ward, A. B., Carlin, A. F., and Esko, J. D. (2020) SARS-CoV-2 Infection Depends on Cellular Heparan Sulfate and ACE2. Cell. https.//doi.org/10.1016/j.cell.2020.09.033 
11. Sarrazin, S., Lamanna, W., and Esko, J. D. (2011) Heparan sulfate proteoglycans. Cold Spring Harb. Perspect. Biol. 3, a004952.

12. Rosenberg, R. D., Shworak, N. W., Liu, J., Schwartz, J. J., and Zhang, L. (1997) Heparan sulfate proteoglycans of the cardiovascular system. Specific structures emerge but how is synthesis regulated? J. Clin. Invest. 99, 2062-2070.

13. Lindahl, U., Kusche-Gullberg, M., and Kjellen, L. (1998) Regulated diversity of heparan sulfate. $J$. Biol. Chem. 273, 24979-24982.

14. Li, J.-P. and Kushe-Gullberg, M. (2016) Heparan Sulfate: Biosynthesis, Structure, and Function. Int. Rev. Cell. Mol. Biol. 325, 215-273.

15. Thacker, B. E., Xu, D., Lawrence, R., and Esko, J. D. (2014) Heparan sulfate 3-O-sulfation: a rare modification in search of a function. Matrix Biol. 35, 60-72.

16. Sankaranarayanan, NV., Bi, Y., Kuberan, B., and Desai, U. R. (2020) Combinatorial virtual library screening analysis of antithrombin binding oligosaccharide motif generation by heparan sulfate 3- OSulfotransferase 1. Comput. Struct. Biotechnol. J. 18, 933-941.

17. Tiwari, V., Clement, C., Duncan, M. B., Chen, J., Liu, J., and Shukla, D. (2004) A role for 3-O-sulfated heparan sulfate in cell fusion induced by herpes simplex virus type 1. J. Gen. Virol. 85, 805-809.

18. O'Donnell, C. D., Tiwari, V., Oh, M.-J., and Shukla, D. (2006) A role for heparan sulfate 3-Osulfotransferase isoform 2 in herpes simplex virus type 1 entry and spread. Virology 346, 452-459.

19. Tiwari, V., O'Donnell, C. D., Oh, M.-J., Valyi-Nagy, T., and Shukla, D. (2005) A role for 3-Osulfotransferase isoform-4 in assisting HSV-1 entry and spread. Biochem. Biophys. Res. Commun. 338, 930-937.

20. Xu, D., Tiwari, V., Xia, G., Clement, C., Shukla, D., and Liu, J. (2005) Characterization of heparan sulphate 3-O-sulphotransferase isoform 6 and its role in assisting the entry of herpes simplex virus type 1. Biochem. J. 385, 451-459.

21. Tiwari, V., Tarbutton, M. S., and Shukla, D. (2015) Diversity of heparan sulfate and HSV entry: basic understanding and treatment strategies. Molecules 20, 2707-2727.

22. Tiwari, V., ten Dam, G. B., Yue, B. Y., van Kuppevelt, T. H., and Shukla, D. (2007) Role of 3-Osulfated heparan sulfate in virus-induced polykaryocyte formation. FEBS Lett. 581, 4468-4472.

23. Tiwari, V., Liu, J., Valyi-Nagy, T., and Shukla, D. (2011) Anti-heparan sulfate peptides that block herpes simplex virus infection in vivo. J. Biol. Chem. 286, 25406-25415.

24. 24 Haines, K. M., Vande Burgt, N. H., Francica, J. R., Kaletsky, R. L., and Bates, P. (2012) Chinese hamster ovary cell lines selected for resistance to ebolavirus glycoprotein mediated infection are defective for NPC1 expression. Virology 432, 20-28.

25. 25 Wang, H., Yang, P., Liu, K., Guo, F., Zhang, Y., Zhang, G., and Jiang, C. (2008) SARS coronavirus entry into host cells through a novel clathrin- and caveolae-independent endocytic pathway. Cell Res. 18, 290-301.

26. Hoffmann, M., Kleine-Weber, H., Krüger, N., Müller, M., Drosten, C., and Pöhlmann, S. (2020) The novel coronavirus 2019 (2019-nCoV) uses the SARS-coronavirus receptor ACE2 and the cellular protease TMPRSS2 for entry into target cells. bioRxiv. 2020:2020.01.31.929042.

27. Kuba, K., Imai, Y., Rao, S., Gao, H., Guo, F., Guan, B., Huan, Y., Yang, P., Zhang, Y., Deng, W., Bao, L., Zhang, B., Liu, G., Wang, Z., Chappell, M., Liu, Y., Zheng, X., Leibbrandt, A., Wada, T., Slutsky, A. S., Liu, D., Qin, C., Jiang, C., and Penninger, J. M. (2005) A crucial role of angiotensin converting enzyme 2 (ACE2) in SARS coronavirus-induced lung injury. Nat. Med. 11, 875-879. 
28. Li, W., Moore, M. J., Vasilieva, N., Sui, J., Wong, S. K., Berne, M. A., Somasundaran, M., Sullivan, J. L., Luzuriaga, K., Greenough, T. C., Choe, H., and Farzan, M. (2003) Angiotensin-converting enzyme 2 is a functional receptor for the SARS coronavirus. Nature 426, 450-454.

29. Ou, X., Liu, Y., Lei, X., Li, P., Mi, D., Ren, L., Guo, L., Chen, T., Hu, J., Xiang, Z., Mu, Z., Chen, X., Chen, J., Hu, K., Jin, Q., Wang, J., and Qian, Z. (2020) Characterization of spike glycoprotein of SARSCoV-2 on virus entry and its immune cross-reactivity with SARS-CoV. Nat. Commun. 11, 1620.

30. Wrapp, D., Wang, N., Corbett, K. S., Goldsmith, J. A., Hsieh, C. L., Abiona, O., Graham, B. S., and McLellan, J. S. (2020) Cryo-EM structure of the 2019-nCoV spike in the prefusion conformation. Science 367, 1260-3.

31. Shang, J., Ye, G., Shi, K., Wan, Y., Luo, C., Aihara, H., Geng, Q., Auerbach, A., and Li, F. (2020) Structural basis of receptor recognition by SARS-CoV-2. Nature 581, 221-224.

32. Yan, R., Zhang, Y., Li, Y., Xia, L., Guo, Y., and Zhou, Q. (2020) Structural basis for the recognition of SARS-CoV-2 by full-length human ACE2. Science 367, 1444-1448.

33. Belouzard, S., Chu, V. C., and Whittaker, G. R. (2009) Activation of the SARS coronavirus spike protein via sequential proteolytic cleavage at two distinct sites. Proc. Natl. Acad. Sci. 106, 5871-5876.

34. Zhang, J., Wan, Y., Luo, C., Ye, G., Geng, Q., Auerbach, A., and Li, F. (2020) Cell entry mechanisms of SARS-CoV-2. Proc. Natl. Acad. Sci. 117, 11727-11734.

35. Sankaranarayanan, NV., Nagarajan, B., and Desai, U. R. (2018) So you think computational approaches to understanding glycosaminoglycan-protein interactions are too dry and too rigid? Think again! Curr. Opin. Struct. Biol. 50, 91-100.

36. Kim, S. Y., Jin, W., Sood, A., Montgomery, D. W., Grant, O. C., Fuster, M. M., Fu, L., Dordick, J. S., Woods, R. J., Zhang, F., and Linhardt, R. J. (2020) Characterization of heparin and severe acute respiratory syndrome-related coronavirus 2 (SARS-CoV-2) spike glycoprotein binding interactions. Antiviral. Res. 181, 104873.

37. Kummarapurugu, A. B., Afosah, D. K., Sankaranarayanan, NV., Navaz Gangji, R., Zheng, S., Kennedy, T., Rubin, B. K., Voynow, J. A., and Desai, U. R. (2018) Molecular principles for heparin oligosaccharide-based inhibition of neutrophil elastase in cystic fibrosis. J. Biol. Chem. 293, 1248012490.

38. Sankaranarayanan, NV., Strebel, T. R., Boothello, R. S., Sheerin, K. J., Raghuraman, A., Salas, F., Mosier, P. D., Watermeyer, N., Oscarson, S., and Desai, U. R. (2017) A Hexasaccharide Containing Rare 2-O-Sulfate-Glucuronic Acid Residues Selectively Activates Heparin Cofactor II. Angew. Chem. Int. Ed. Engl. 56, 2312-2317.

39. Chen, J., Duncan, M. B., Carrick, K., Pope, R. M., and Liu, J. (2003) Biosynthesis of 3-O-sulfated heparan sulfate: unique substrate specificity of heparan sulfate 3-O-sulfotransferase isoform 5. Glycobiology 13, 785-794.

40. Desai, U. R., Wang, H. M., and Linhardt, R. J. (1993) Specificity studies on the heparin lyases from Flavobacterium heparinum. Biochemistry 32, 8140-8145.

41. Elste, J., Kaltenbach, D., Patel, V. R., Nguyen, M. T., Sharthiya, H., Tandon, R., Mehta, S. K., Volin, M. V., Fornaro, M., Tiwari, V., and Desai, U. R. (2020) Inhibition of Human Cytomegalovirus Entry into Host Cells Through a Pleiotropic Small Molecule. Int. J. Mol. Sci. 21, 1676.

42. Gallegos, K. M., Taylor, C. R., Rabulinski, D. J., Del Toro, R., Girgis, D. E., Jourha, D., Tiwari, V., Desai, U. R., and Ramsey, K. H. (2019) A Synthetic, Small, Sulfated Agent Is a Promising Inhibitor of Chlamydia spp. Infection in vivo. Front. Microbiol. 9, 3269. 
43. Majmudar, H. , Hao, M. , Sankaranarayanan, NV., Zanotti, B., Volin, M. V., Desai, U. R., and Tiwari, V. (2019) A synthetic glycosaminoglycan mimetic blocks HSV-1 infection in human iris stromal cells. Antiviral Res. 161, 154-162.

44. Gangji, R. N., Sankaranarayanan, NV., Elste, J., Al-Horani, R. A., Afosah, D. K., Joshi, R., Tiwari, V., and Desai, U. R. (2018) Inhibition of Herpes Simplex Virus-1 Entry into Human Cells by Nonsaccharide Glycosaminoglycan Mimetics. ACS Med. Chem. Lett. 9, 797-802.

45. Dull, T., Zufferey, R., Kelly, M., Mandel, R. J., Nguyen, M., Trono, D., and Naldini, L. (1998) A thirdgeneration lentivirus vector with a conditional packaging system. J. Virol. 72, 8463-8471.

46. Zang, R., Castro, M. F. G., McCune, B. T., Zeng, Q., Rothlauf, P. W., Sonnek, N. M., Liu, Z., Brulois, K. F., Wang, X., Greenberg, H. B., Diamond, M. S., Ciorba, M. A., Whelan, S. P. J., and Ding, S. (2020) TMPRSS2 and TMPRSS4 promote SARS-CoV-2 infection of human small intestinal enterocytes. Sci. Immunol. 5, eabc3582.

47. Schiffner, T., Sattentau, Q. J., and Duncan, C. J. A. (2013) Cell-to-cell spread of HIV-1 and evasion of neutralizing antibodies. Vaccine 31, 5789-5797.

48. Haeger, S. M., Yang, Y., and Schmidt, E. P. (2016) Heparan Sulfate in the Developing, Healthy, and Injured Lung. Am. J. Respir. Cell. Mol. Biol. 55, 5-11.

49. Cao, C., Huang, L., Liu, K., Ma, K., Tian, Y., Qin, Y., Sun, H., Ding, W., Gui, L., and Wu, P. (2020) Amino acid variation analysis of surface spike glycoprotein at 614 in SARS-CoV-2 strains. Genes Dis. DOI: $10.1016 /$ j.gendis.2020.05.006.

50. Sobhy, H. (2017) A comparative review of viral entry and attachment during large and giant dsDNA virus infections. Arch. Virol. 162, 3567-85.

51. Mycroft-West, C., Su, D., Elli, S., Li, Y., Guimond, S., Miller, G., Turnbull, J., Yates, E., Guerrini, M., Fernig, D., Lima, M., and Skidmore, M. (2020) The 2019 coronavirus (SARS-CoV-2) surface protein (Spike) S1 Receptor Binding Domain undergoes conformational change upon heparin binding. BioRxiv https.//doi.org/10.1101/2020.02.29.971093

52. Kaltenbach, K. D., Jaishankar, D., Hao, M., Beer, J. C., Volin, M. V., Desai, U. R., and Tiwari, V. (2018) Sulfotransferase and Heparanase: Remodeling Engines in Promoting Virus Infection and Disease Development. Front. Pharmacol. 9, 1315.

53. Liu, L., Chopra, P., Li, X., Wolfert, M. A., Tompkins, S. M., and Boons, G.-J. (2020) SARS-CoV-2 spike protein binds heparan sulfate in a length- and sequence-dependent manner. BioRxiv https.//doi.org/10.1101/2020.05.10.087288

54. Guimond, S. E., Mycroft-West, C. J., Gandhi, N. S., Tree, J. A., Buttigieg, K. R., Coombes, N., Nystrom, K., Said, J., Setoh, Y. X., Amarilla, A., Modhiran, N., Sng, D. J. J., Chhabra, M., Watterson, D., Young, P. R., Khromykh, A. A., Lima, M. A., Fernig, D. G., Su, D., Yates, E. A., Hammond, E., Dredge, K., Carroll, M. W., Trybala, E., Bergstrom, T., Ferro, V., Skidmore, M. A., and Turnbull, J. E. (2020) Pixatimod (PG545), a clinical-stage heparan sulfate mimetic, is a potent inhibitor of the SARSCoV-2 virus. BioRxiv https.//doi.org/10.1101/2020.06.24.169334

55. da Silva, C. S. B., Thaler, M., Tas, A., Ogando, N. S., Bredenbeek, P. J., Ninaber, D. K., Wang, Y., Hiemstra, P. S., Snijder, E. J., and van Hemert, M. J. (2020) Suramin Inhibits SARS-CoV-2 Infection in Cell Culture by Interfering with Early Steps of the Replication Cycle. Antimicrob. Agents Chemother. 64, e00900-20.

56. Al-Horani, R. A., Ponnusamy, P., Mehta, A. Y., Gailani, D., and Desai, U. R. (2013) Sulfated pentagalloylglucoside is a potent, allosteric, and selective inhibitor of factor Xia. J. Med. Chem. 56, 867-878. 

available under aCC-BY-NC-ND 4.0 International license.

57. Kipshidze, N., Dangas, G., White, C. J., Kipshidze, N., Siddiqui, F., Lattimer, C. R., Carter, C. A., and Fareed, J. (2020) Viral Coagulopathy in Patients With COVID-19: Treatment and Care. Clin. Appl. Thromb. Hemost. (In press). DOI:10.1177/1076029620936776 


\section{Figure Legends}

Figure 1. Selective recognition of 3-O-sulfated heparan sulfate (HS) by SgP of SARS-CoV-2 in promotion of cell fusion. (A) "Effector" CHO-K1 cells expressed SgP of SARS-CoV-2, while the "target" CHO-K1 cells expressed 3OST-3 negative control CHO-K1 cells expressing neither 3OST-3 $3_{\mathrm{B}}$ nor ACE-2 receptor. (B) Microscopic visualization of syncytia induced by SgP-HSPG or SgP-ACE-2 interaction. (C) Comparison of cell fusion when "target" HSPGs carry either 3OST-3 B or 3OST-5 generated sequences. Error bars $= \pm 1 \mathrm{SD} ; * * \mathrm{P}<0.05$, one-way ANOVA.

Figure 2. Computational screening of a library of di-, tetra- and hexa- saccharide sequences of HS (total 27,930 topologies) against RBD of SgP for identification of origin of selectivity at the atomistic level. (A) The plausible sites of HS binding onto SgP trimer include ${ }^{453}$ YRLFRKS $^{459}$ (yellow $=$ RBD), ${ }^{681}$ PRRARS ${ }^{686}$ (red); and ${ }^{810}$ SKPSKRS $^{816}$ (orange) in the trimeric SgP (chains A, B, and $\mathrm{C}$ are shown in pink, grey and blue, respectively). (B) The dual-filter algorithm used to identify high affinity and high specificity HS topologies that bind to the RBD of SgP. GOLDscore was the first filter, while RMSD (consistency of binding) was the second filter. (C) Results after the first filter in the form of a histogram of the number of HS hexasaccharide topologies for every 10 unit change in GOLDscore. Inset shows promising high affinity topologies. (D) The zoomed version of the two high selectivity HS hexasaccharides binding to the RBD of SgP (sticks in green and cyan color). See Figure S4 for details on the structure of these sequences and their nature of interaction with residues constituting the RBD of SgP. (E) Overlay showing the two HS hexasaccharides (shown in van der Waals rendering) binding in the RBD domain of SgP (trimer) in relationship with the known ACE2 (green ribbons) site of binding from the cryoEM structure (PDB:6M0J).

Figure 3. Antagonism of 3-O-sulfated HS receptor through (A) either cleavage of HSPGs by heparinase I (Hep 1, 1.5 units/ml), competitive inhibition with generic $(10 \mu \mathrm{M})$ or 3-O-sulfate-specific (10 $\mu \mathrm{M})$ peptides, or (B) competition with 3-O-sulfate containing synthetic pentasaccharide, fondaparinux. "Effector" CHO-K1 cells expressed SgP of SARS-CoV-2, while "target" CHO$\mathrm{K} 1$ cells expressed $3 \mathrm{OST}-3_{\mathrm{B}}$. Wild-type HS indicated by (-) refer to "target" negative control CHO-K1 cells devoid of 3 OST $-3_{\mathrm{B}}$ expression. Error bars $= \pm 1 \mathrm{SD}$; ** $\mathrm{P}<0.05$, one-way ANOVA.

Figure 4. Antagonism of SgP-mediated cell fusion by SPGG, a synthetic, small, highly sulfated compound in two different systems. (A) Inhibition of cell-to-cell fusion between "effector" CHO-K1 cells expressing SgP of SARS-CoV-2 and "target" 293T cells (A) or CHO-K1 cells (B). Wild-type HS expressing in in both cell types represents the negative control (shown as $(-)$ ). Positive control 293T cells (shown as (+)) express ACE-2 receptor, while that in the CHO$\mathrm{K} 1$ cells express $3 \mathrm{OST}-3_{\mathrm{B}}$ enzyme, which modifies surface HSPGs to 3-O-sulfated HS. Error bars $= \pm 1 \mathrm{SD} ; * * \mathrm{P}<0.05$, one-way ANOVA. (C) Inhibition of SARS-CoV-2 pseudo typed lentivirus with SPGG. Treatment of either the pseudo-virus (mode A, blue) or 293T cells (mode $\mathrm{B}$, red) with SPGG reduced viral entry (measured $48 \mathrm{~h}$ post-transduction) using GFPfluorescence of the pseudo-virus. Error bars $= \pm 1$ SD. Grey dotted line represents $50 \%$ inhibition. D) Representative fluorescence microscopy of the mock-treated (panel (i)) or SPGG-treated 293T cells (panel (ii)) at 10X magnification. (E) Structure of SPGG. 
A
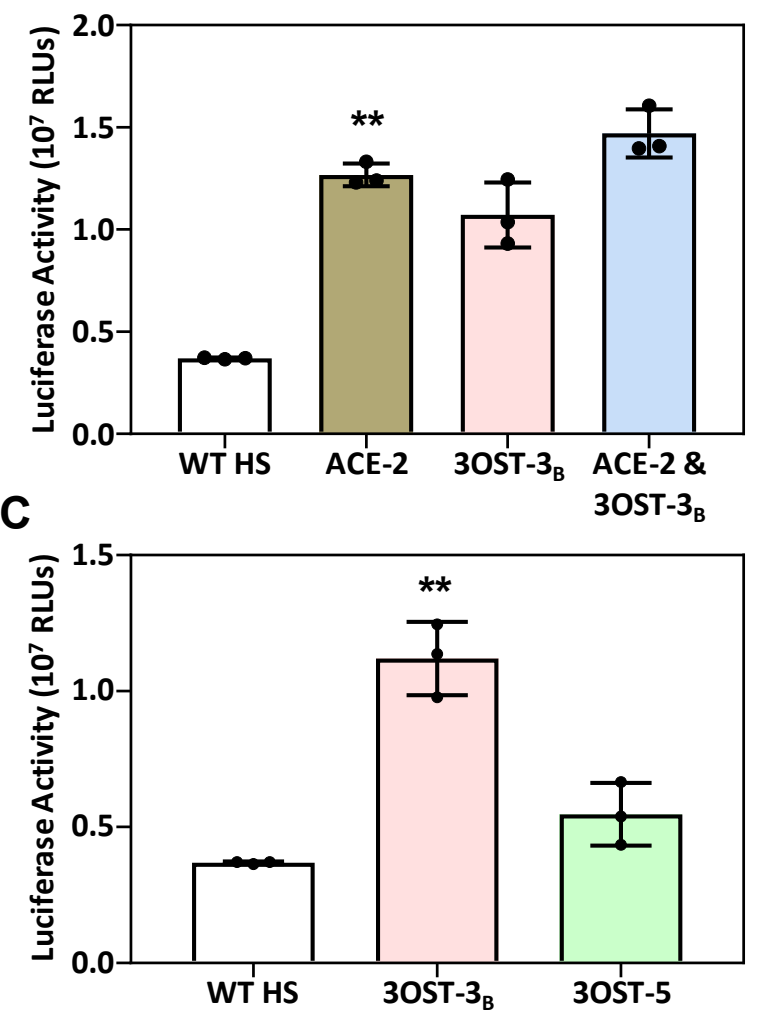

B
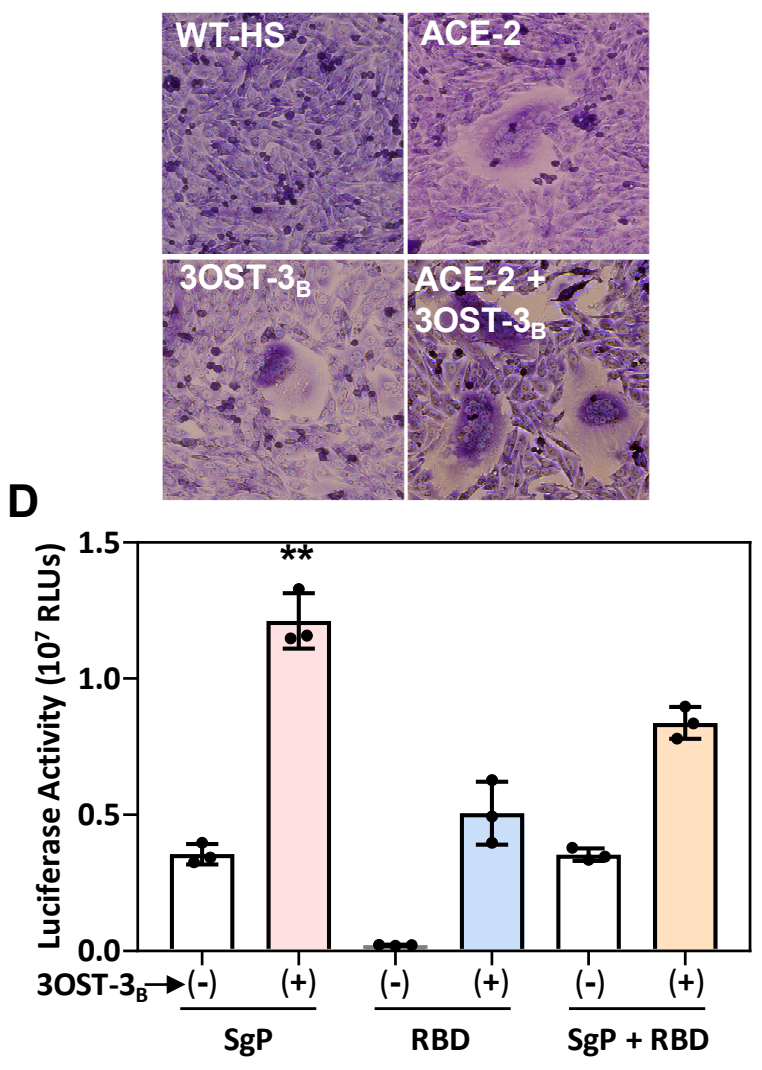

Figure 1 

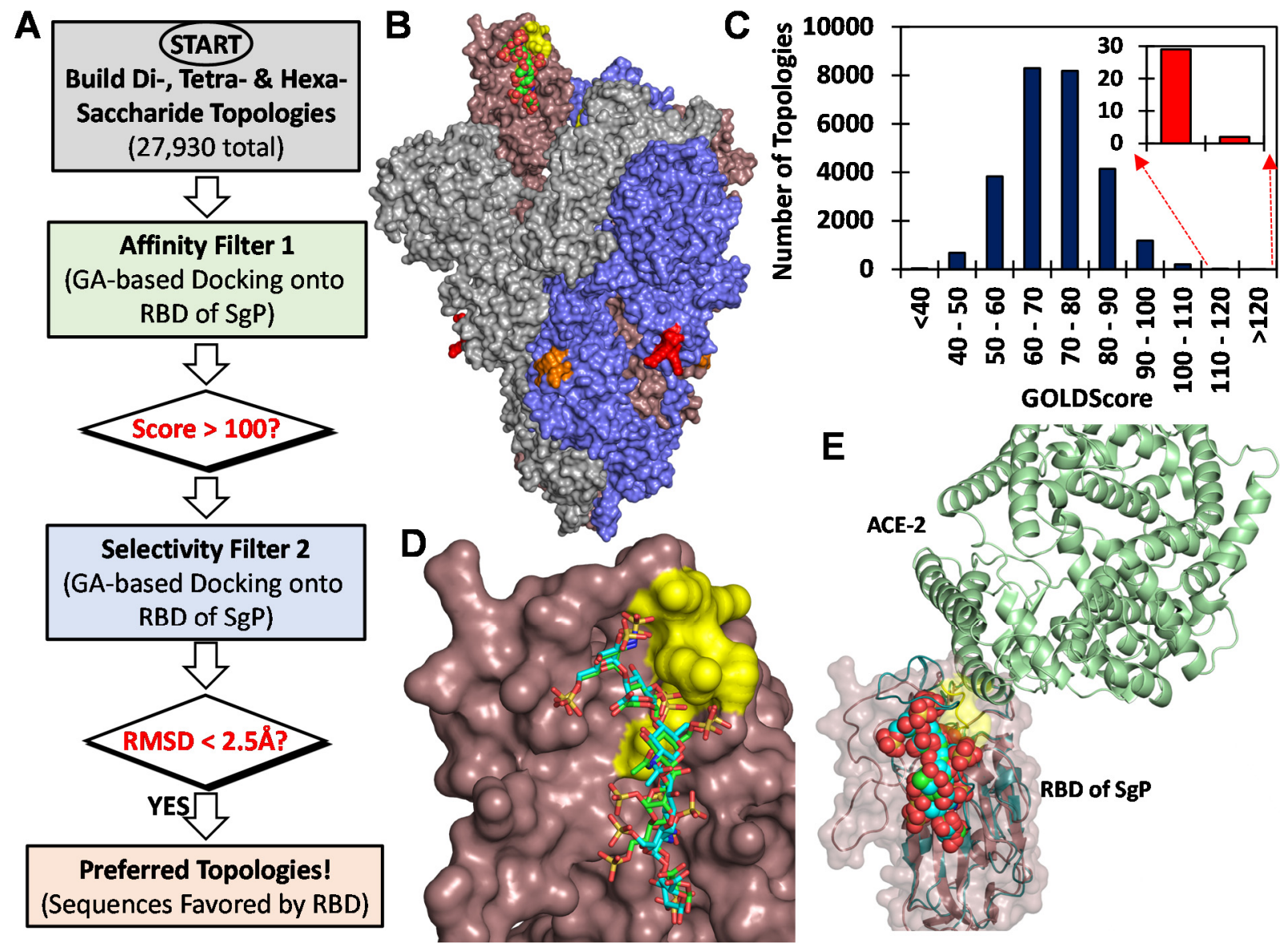

Figure 2 
A

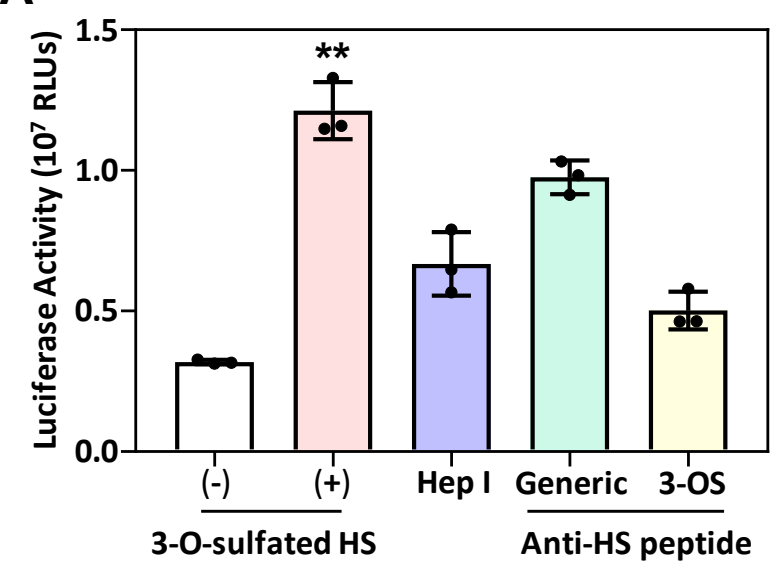

B

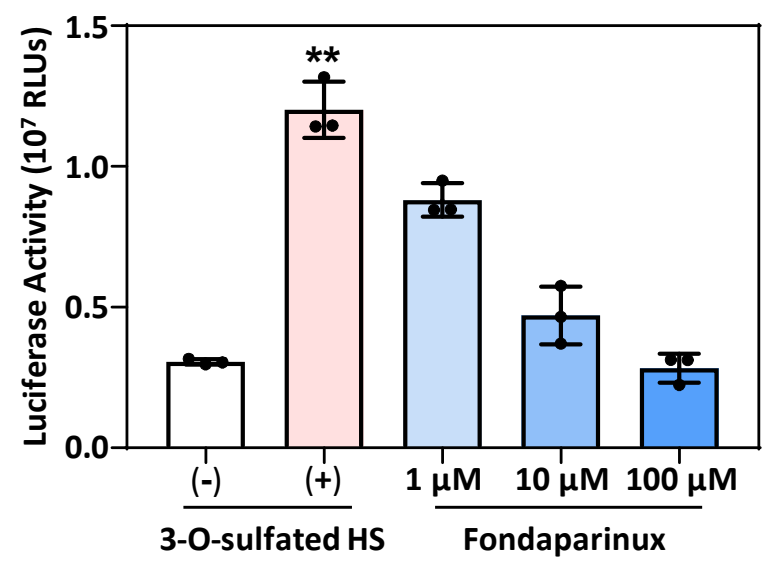

Figure 3 
A
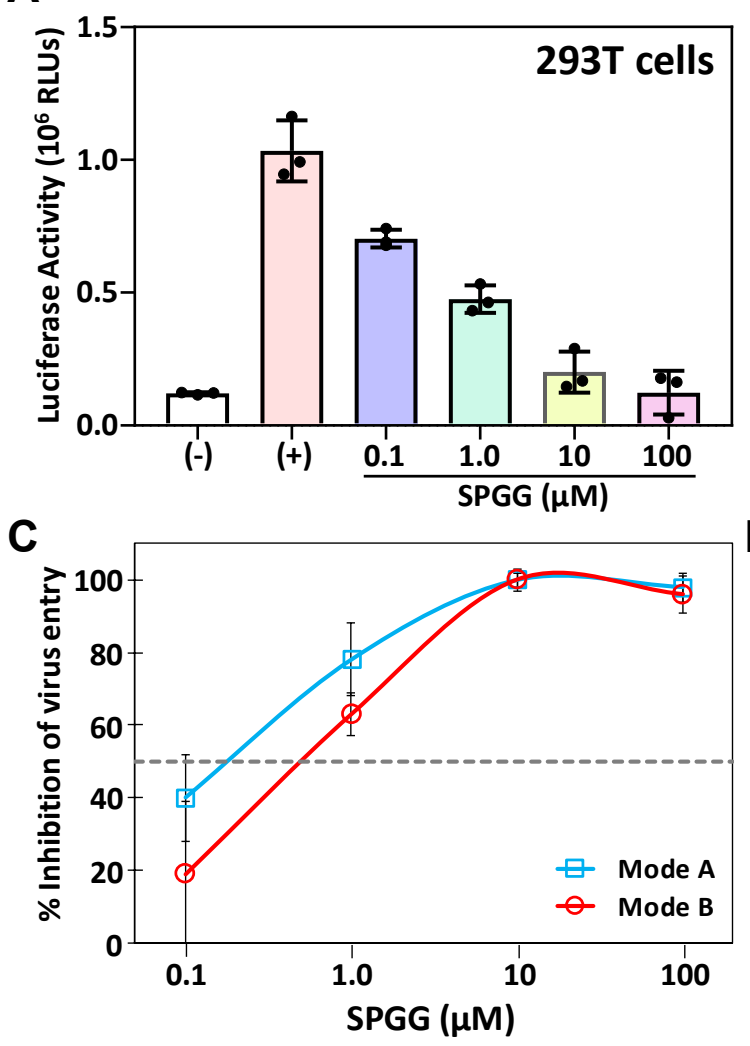

B
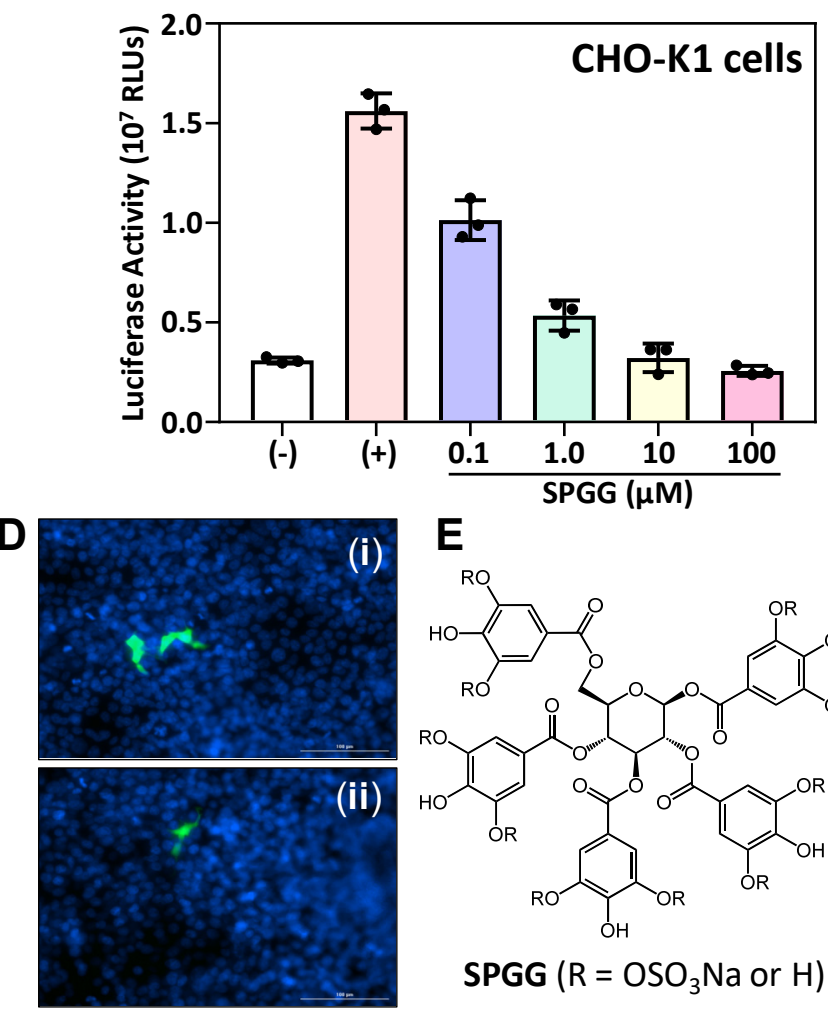

E

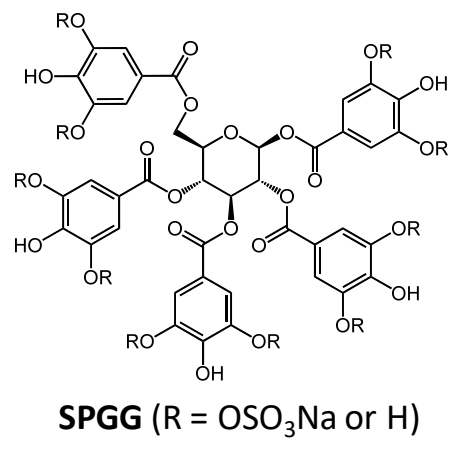

Figure 4 